\section{pGSTp: An IVET- Compatible Promoter Probe Vector Conferring Resistance to Trimethoprim}

BioTechniques 29:954-958 (November 2000)

Promoter probe plasmids that can integrate into the chromosome via homologous recombination are an important tool in studying gene regulation. These vectors have proved useful in identifying bacterial genes that are specifically expressed during infection of cultured cells or animal models. A common system that is used is in vivo expression technology (IVET) (9). The original IVET-based vectors involve cloning random chromosomal DNA fragments upstream of a promoterless purA gene (9).

Bacterial genes, which are active during infection, are identified by the ability of their cognate promoters to effect the complementation of a purA auxotroph. One disadvantage of these systems is the requirement for a bacterial strain containing a nonrevertable auxotrophic mutation. This potential drawback can be circumvented by using a promoterless antibiotic resistance gene and selecting for bacterial co-integrate clones that resist antibiotic challenge during infection (10). A promoterless chloramphenicol acetyltransferase (cat) gene has been found to be useful in this respect, especially for studying pathogens that can adopt an intracellular lifestyle, as chloramphenicol can penetrate mammalian cells $(10,16)$. Plasmids such as pIVET8 and pGY2 $(10,16)$, which contain the bla gene (and also the aadA gene in the case of pGY2), are only useful in Gram-negative bacteria that are sensitive to $\beta$-lactams and streptomycin. However, many pathogenic bacteria, such as members of the genus Burkholderia, exhibit high levels of resistance to both antibiotics. Here, we describe the construction and use of a promoter probe suicide vector that contains a trimethoprim resistance marker, which allows selection in many bacteria that are naturally resistant to most $\beta$ lactams and aminoglycosides.
The promoter probe vector $\mathrm{pKK} 232$ 8 (Amersham Pharmacia Biotech, Little Chalfont, UK) contains a 660-bp promoterless cat gene preceded by stop codons in all three reading frames (1). Using oligonucleotide primers designed to incorporate single $B a m \mathrm{HI}, K p n \mathrm{I}$, SmaI, BglII and XbaI sites upstream of cat and a restriction site for XhoI downstream of $c a t$, the complete polypeptide coding sequence, together with its cognate ribosome binding site but excluding the stop codons, was amplified by PCR (13). The primers used were: catf,
5'-GCGGGATCCGGTACCCGGGAG ATCTTCTAGAAGGAGCTA $\underline{\text { AGGAA }}$ GCTAA-3' and catr, 5'-GCGCTCGA GCACTTATTCAGGCGTAGC-3' (cat Shine-Dalgarno and stop codon sequences are underlined). The 880-bp PCR product was digested with $B a m H I$ and XhoI and cloned between the compatible $B g l I I$ and SalI sites of the mobilizable suicide vector pUTmini-Tn5Cm (3) to create pGS18. This step resulted in substitution of the promoterless cat cassette for the mini-Tn5Cm transposon and adjacent tnp gene.

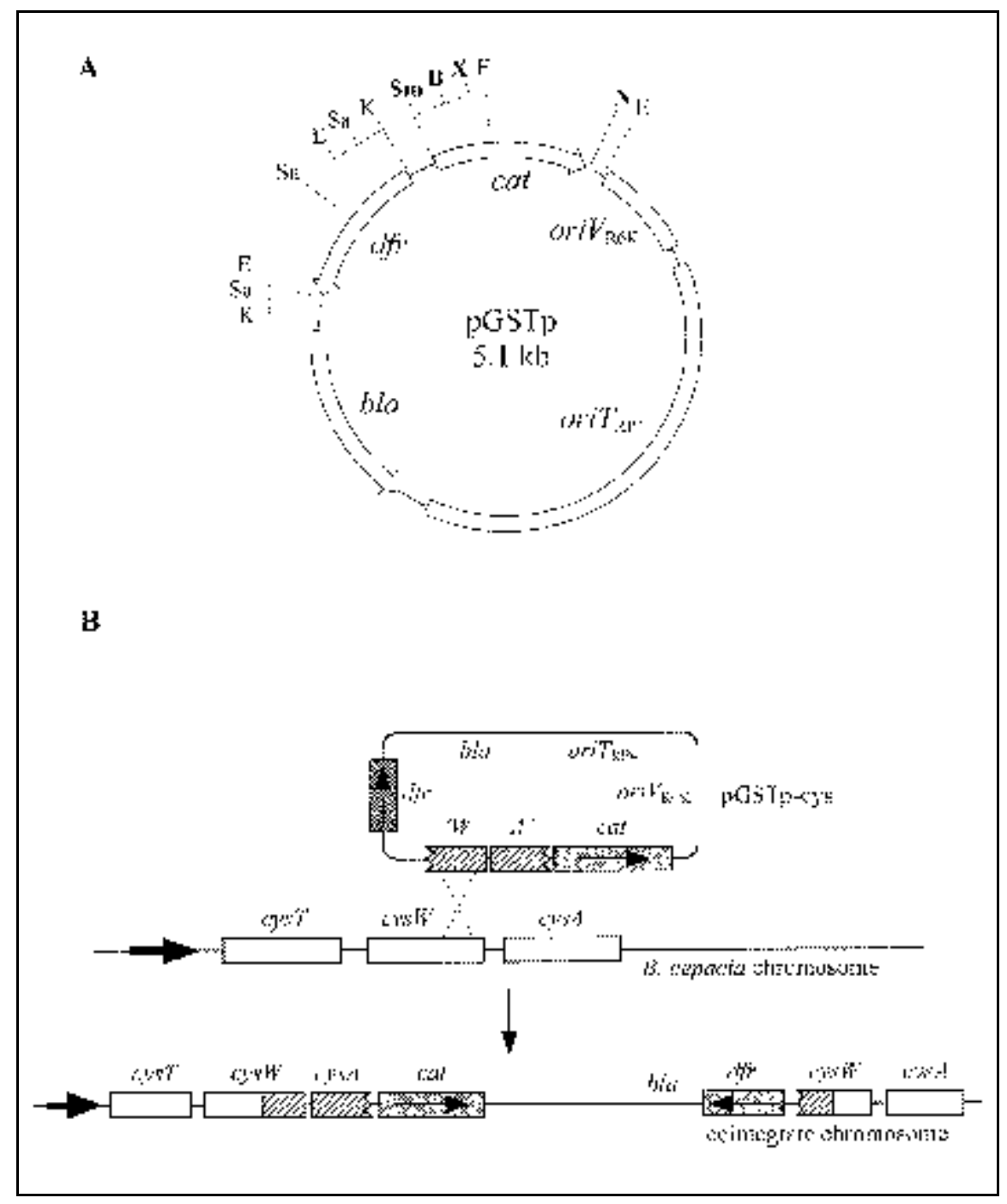

Figure 1. Construction and use of pGSTp. (A) Map of the promoter probe suicide plasmid pGSTp. Abbreviations: cat, promoterless chloramphenicol acetyltransferase gene; bla, $\beta$-lactamase gene; $d f r$, dihydrofolate reductase gene; ori $V_{\mathrm{R} 6 \mathrm{~K}}$, origin of replication from plasmid $\mathrm{R} 6 \mathrm{~K} ;$ oriT $_{\mathrm{RP} 4}$, origin of conjugal transfer from plasmid RP4; B, BglII; E, EcoRI; K, KpnI; N, NotI; Sa, SacI; Sm, SmaI; X, XbaI. Unique restriction sites are shown in bold. (B) Integration of pGSTp-cys into the genome of B. cepacia occurs through a single crossover recombination event (dotted lines) between the plasmid-borne cys DNA fragment and homologous sequences on the B. cepacia chromosome. The cys promoter located upstream of cys $T$ is indicated by a thick arrow. The orientation of the cat and $d f r$ genes are shown. 
Although pGS18 confers resistance to $\beta$-lactams, to permit selection of this plasmid in a wide range of Gram-negative bacteria, an additional antibiotic marker was added. p34E-Tp (2) was digested with $K p n I$ to release the $d f r$ gene, encoding resistance to trimethoprim, which was then cloned into the KpnI site of pGS18. Plasmid clones were selected in which the trimethoprim cassette was inserted in the opposite transcriptional orientation to cat, thus preventing read-through transcription into cat from the $d f r$ promoter. One such derivative, pGSTp, is shown in Figure 1A. pGSTp contains the origin of replication of R6K and can therefore only be maintained as an extrachromosomal element in hosts harboring a copy of the pir gene (encoding the $\pi$ replication protein) (4). However, derivatives of this plasmid, which contain cloned bacterial chromosomal DNA, can be maintained in bacteria that are devoid of pir by integration into the host chromosome following recombination between homologous sequences on the plasmid and on the bacterial chromosome. The vector also contains the conjugal origin of transfer of RP4, which facilitates mobilization of the plasmid to other Gram-negative bacte- ria when the RP4 mobilization functions are provided in trans (15).

To examine the usefulness of this vector for promoter analysis, we used it to monitor the regulation of the cysT, cys $W$ and cysA genes of Burkholderia cepacia, which were recently identified in our laboratory (K.L. Farmer and M.S. Thomas, unpublished observations). These genes are functional homologues of the cysTWA genes of $E$. coli, which encode an $\mathrm{ABC}$ transporter (sulfate permease) involved in sulfate uptake $(6,8)$. In E. coli, these genes are induced by sulfur limitation, whereas L-cysteine acts indirectly as an anti-inducer of cys regulon expression (5). A $1.2-\mathrm{kb}$ HindIII-SmaI fragment from the B. cepacia genome, containing a $3^{\prime}$ segment of cys $W$ and a $5^{\prime}$ segment of the adjacent $c y s A$ gene, was filled-in with DNA polymerase I Klenow fragment (Life Technologies, Paisley, UK) and cloned into the SmaI site of pGSTp in the same orientation as cat (Figure 1B). The resultant plasmid, pGSTp-cys, was mobilized into B. cepacia $715 \mathrm{j}$ (11) by conjugation using E. coli BW19851 (12). B. cepacia exconjugants harboring pGSTp-cys were selected on Iso-sensitest agar (Oxoid, Basingstoke, UK) containing trimethoprim $(50 \mu \mathrm{g} /$

1

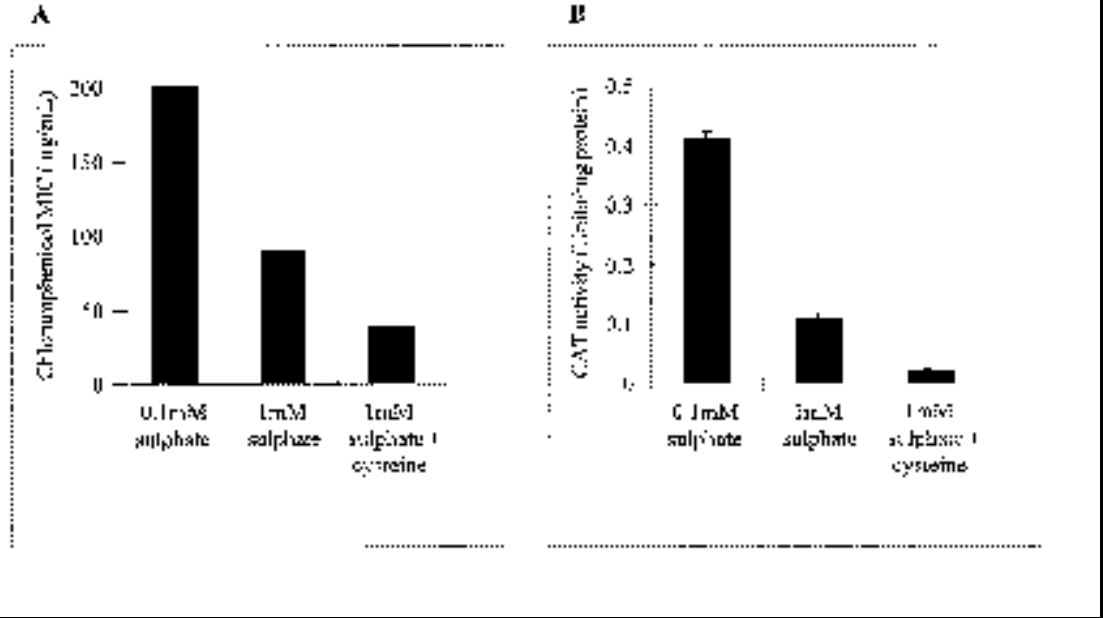

Figure 2. Use of pGSTp to monitor cys promoter activity. Effect of sulfur availability on the MIC for chloramphenicol and CAT activity of a B. cepacia pGSTp-cys co-integrate strain. (A) The MIC of chloramphenicol for the co-integrate strain was determined following growth on M9-glucose agar containing $1 \mathrm{mM}$ sulfate, $0.1 \mathrm{mM}$ sulfate or $1 \mathrm{mM}$ sulfate plus cysteine $(40 \mu \mathrm{g} / \mathrm{mL})$ in the presence of increasing concentrations of chloramphenicol. (B) The co-integrate strain was grown at $37^{\circ} \mathrm{C}$ in M9-glucose medium containing $1 \mathrm{mM}$ sulfate, $0.1 \mathrm{mM}$ sulfate or $1 \mathrm{mM}$ sulfate plus cysteine. CAT assays were performed in triplicate on sonicated cells. One unit of CAT activity is equal to $1 \mu \mathrm{mol}$ of chloramphenicol acetylated per minute at $37^{\circ} \mathrm{C}$.
$\mathrm{mL})$ and kanamycin $(50 \mu \mathrm{g} / \mathrm{mL})$ and appeared at a frequency of $2 \times 10^{-6}$ per recipient. Using the trimethoprim resistance cassette as a probe, Southern blotting was carried out on genomic DNA purified from 12 randomly selected exconjugants. The results (not shown) demonstrated that in all cases, the plasmid had integrated at the cysA locus of the $715 \mathrm{j}$ genome, placing cat under control of the cys promoter (Figure 1B).

The usefulness of this vector for promoter analysis was examined in two ways. First, we confirmed that cat expression was under cys promoter control by demonstrating that the chloram phenicol resistance of the co-integrate strains was regulated according to sulfate availability (Figure 2A). Thus, the minimum inhibitory concentration (MIC) of 715j (pGSTp-cys) for chloramphenicol on M9 minimal agar containing glucose is $90 \mu \mathrm{g} / \mathrm{mL}$ (the sulfate content in standard M9 medium is 1 $\mathrm{mM}$, supplied in the form of $\mathrm{MgSO}_{4}$ ), whereas on M9 agar containing a low concentration of sulfate $(0.1 \mathrm{mM})$, the MIC for chloramphenicol was 200 $\mu \mathrm{g} / \mathrm{mL}$ (the magnesium ion content was maintained at $1 \mathrm{mM}$ by supplementation with $0.9 \mathrm{mM} \mathrm{MgCl} 2$ ). In contrast, when standard M9 minimal agar was supplemented with cysteine $(40 \mu \mathrm{g} /$ $\mathrm{mL}$ ), which bypasses the requirement for exogenously added sulfate, the MIC for chloramphenicol was the same as the parent strain $(40 \mu \mathrm{g} / \mathrm{mL})$.

In a second experiment, the variation in transcriptional activity of $c y s A$ in response to exogenously added sulfate was quantitated by measuring CAT activity in $715 \mathrm{j}$ cells harboring the pGSTp-cys co-integrate $(7,14)$. When cells were grown in standard M9 medium, CAT activity was $0.11 \mathrm{U}$. Further inducing cys promoter activity by reducing the level of sulfate to $0.1 \mathrm{mM}$ resulted in an increase in CAT activity to $0.41 \mathrm{U}$. When cysteine was added to standard M9 media, cat expression was repressed 20-fold (CAT activity 0.02 $\mathrm{U})$ relative to fully induced levels (Figure 2B). The results indicate that, as in E. coli, the activity of the cysTWA locus in $B$. cepacia is regulated by the availability of sulfur.

In conclusion, we have constructed a more versatile mobilizable promoter probe suicide vector for the generation 
of chromosomal transcriptional fusions in Gram-negative bacteria that are sensitive to trimethoprim. These include most members of the Enterobacteriaceae, such as Escherichia, Klebsiella and Proteus. However, it is particularly useful in studies on those Gram-negative bacteria that exhibit low-level resistance to trimethoprim but high resistance to other commonly used antibiotics, such as members of the genus Burkholderia (particularly the human pathogens $B$. cepacia and $B$. pseudomallei) and the opportunist pathogen Stenotrophomonas maltophila (formerly Xanthomonas maltophila). The resultant single-copy fusions are more desirable for studying gene regulation because they avoid problems associated with multicopy systems such as the titration of regulatory proteins. This vector can also be adapted for the identification of bacterial promoters that are specifically expressed in an animal host or within cultured cell lines. The unique NotI site downstream of the promoterless cat gene facilitates the insertion of an additional reporter gene or antibiotic resistance marker as required.

\section{REFERENCES}

1.Brosius, J. 1984. Plasmid vectors for the selection of promoters. Gene 27:151-160.

2.DeShazer, D. and D.E. Woods. 1996. Broadhost range cloning vectors based on the R388 trimethoprim resistance gene. BioTechniques 20:762-764.

3.DeLorenzo, V., M. Herrero, U. Jakubzik and K.N. Timmis. 1990. Mini-Tn5 transposon derivatives for insertion mutagenesis, promoter probing, and chromosomal insertion of cloned DNA in Gram-negative eubacteria. J. Bacteriol. 172:6568-6572.

4.Kolter, R., M. Inuzuka and D.R. Helinski. 1978. Trans-complementation-dependent replication of a low molecular weight origin fragment from plasmid R6K. Cell 15:1199-1208.

5.Kredich, N.M. 1992. The molecular basis for positive regulation of cys promoters in Salmonella typhimuriumand Escherichia coli. Mol. Microbiol. 6:2747-2753.

6.Kredich, N.M. 1996. Biosynthesis of cysteine, p. 514-527. In F.C. Neidhardt (Ed.). E. coli and Salmonella: Cellular and Molecular Biology, 2nd ed., Vol. 1. ASM Press, Washington DC.

7.Li, S.C., C.L. Squires and C. Squires. 1984. Antitermination of $E$. coli rRNA transcription is caused by a control region segment containing lambda nut-like sequences. Cell 38:851860.

8.Linton, K.J. and C.F. Higgins. 1998. The Escherichia coli ATP-binding cassette (ABC) proteins. Mol. Microbiol. 28:5-13.

9.Mahan, M.J., J.M. Slauch and J.J. Mekalanos. 1996. Selection of bacterial virulence genes that are specifically induced in host tissues. Science 259:686-688.

10.Mahan, M.J., J.W. Tobias, J.M. Slauch, P.C. Hanna, R.J. Collier and J.J. Mekalanos. 1995. Antibiotic-based selection for bacterial genes that are specifically induced during infection of a host. Proc. Natl. Acad. Sci. USA 92:669-673.

11.McKevitt, A.I., S. Bajaksouzian, J.D. Klinger and D.E. Woods. 1989. Purification and characterization of an extracellular protease from Pseudomonas cepacia. Infect. Immun. 57:771-778.

12.Metcalf, W.W., W. Jiang and B.L. Wanner. 1994. Use of the rep technique for allele replacement to construct new Escherichia coli hosts for maintenance of R6Ky origin plasmids at different copy numbers. Gene 138:17.

13.Mullis, K.B. and F.A. Faloona. 1987. Specific synthesis of DNA in vitro via a polymerasecatalyzed chain reaction. Methods Enzymol. 155:335-350.

14.Shaw, W.V. 1975. Chloramphenicol acetyltransferase from chloramphenicol-resistant bacteria. Methods Enzymol. 43:737-755.

15.Simon, R., U. Priefer and A. Puhler. 1983. A broad host range mobilization system for in vivo genetic engineering: transposon mutagenesis in Gram negative bacteria. BioTechnology 1:784-791.

16. Young, G.M. and V.L. Miller. 1997. Identification of novel chromosomal loci affecting Yersinia enterocolitica pathogenesis. Mol. Microbiol. 25:319-328.

We thank Kenneth N. Timmis for providing pUTmini-Tn5Cm and Donald E. Woods for providing $\mathrm{p} 34 \mathrm{E}-\mathrm{Tp}$. This work was supported by a studentship from the Division of Molecular and Genetic Medicine, University of Sheffield Medical School. Address correspondence to Dr. Mark S. Thomas or Dr. Jonathan G. Shaw, Division of Molecular and Genetic Medicine, F Floor, University of Sheffield Medical School, Beech Hill Road, Sheffield S10 2RX, UK. e-mail: m.s. thomas@shef.ac.ukorj.g.shaw@shef.ac.uk

Received 12 April 2000; accepted 17 July 2000.

Gil Shalom, Jonathan G. Shaw and Mark S. Thomas University of Sheffield Medical School

Sheffield, UK

\section{$3^{\prime}$ Overhangs Influence PCR-SSCP Patterns}

\author{
BioTechniques 29:958-962 (November 2000)
}

Single strand conformation polymorphism (SSCP) analysis has become a popular method for detecting sequence variation in PCR-amplified DNA. It is a highly sensitive technique that is capable of detecting single-base substitutions under optimized conditions (3). DNA amplified by DNA polymerases, which lack $3^{\prime} \rightarrow 5^{\prime}$ exonuclease (proofreading) activity, often has an additional nontem plate-directed nucleotide added at the $3^{\prime}$ ends (1). The addition of the extra nucleotide is variable, and the nucleotide added is dependent on the $3^{\prime}$ base existing at the blunt end (Table 1). Therefore, PCR products amplified by a DNA polymerase that exhibits extendase activity often consist of a mixture of blunt-ended DNA fragments and those with $3^{\prime}$ overhangs. The presence of these $3^{\prime}$ overhangs may affect the single strand conformation of PCR products and hence change the migration of bands on an SSCP gel. Furthermore, any factor that changes the addition of the $3^{\prime}$ extra nucleotides or causes degradation of the $3^{\prime}$ overhangs may alter the PCR-SSCP pattern. In this study, we tested the effect of the 3' overhangs generated by Taq DNA polymerase (Qiagen $\mathrm{GmbH}$, Hilden, Germany) on SSCP banding patterns.

A fimbrial subunit gene (fimA) of Dichelobacter nodosus was amplified using the blunt end DNA polymerase, Pwo (Roche Molecular Biochemicals, Mannheim, Germany). After purification, half of the PCR product was treated with Taq DNA polymerase and then subjected to SSCP analysis. The PCR product treated with Taq DNA polymerase generated a different SSCP pattern from the untreated product (Figure 1a) and caused a reduction in the mobility of the slowest moving band. This mobility shift can be most clearly seen in Figure 1a, lane 2, where PCR products treated with and without Taq DNA polymerase were mixed and run together in the same lane. As Pwo DNA polymerase produces blunt-ended PCR products, the difference in migration pattern observed is thought to be the di- 